\title{
Fast pulsed radar reflectometry for the Textor Tokamak
}

C. A. J. Hugenholtz, A. J. H. Donné, B. S. Q. Elzendoorn, J. C. van Gorkom, W. Kooijman, H. A. van der Laan, M. J. van de Pol, A. J. Putter, H. J. F. van Ramele, D. Smit, P. C. de Vries, F. Wijnoltz, W. Pysik, G. Waidmann, and G. P. Ermak

Citation: Review of Scientific Instruments 70, 1034 (1999);

View online: https://doi.org/10.1063/1.1149426

View Table of Contents: http://aip.scitation.org/toc/rsi/70/1

Published by the American Institute of Physics

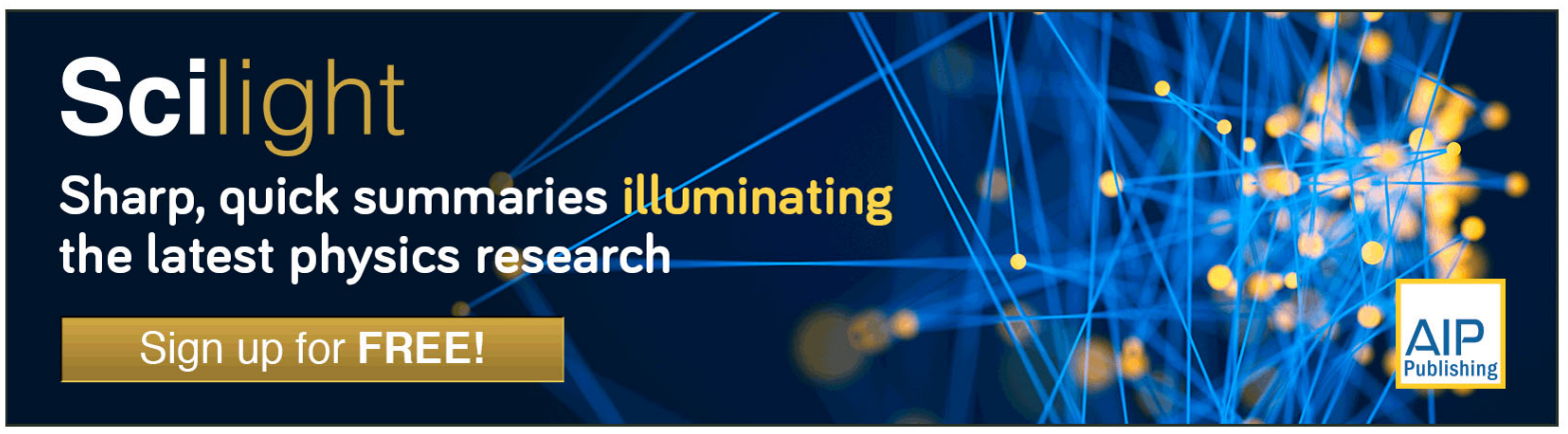




\title{
Fast pulsed radar reflectometry for the Textor Tokamak
}

\author{
C. A. J. Hugenholtz, A. J. H. Donné, B. S. Q. Elzendoorn, J. C. van Gorkom, W. Kooijman, \\ H. A. van der Laan, M. J. van de Pol, A. J. Putter, H. J. F. van Ramele, D. Smit, \\ P. C. de Vries, and F. Wijnoltz \\ FOM Instituut voor Plasmafysica Rijnhuizen, Association EURATOM-FOM, 3430 BE Nieuwegein, \\ The Netherlands \\ W. Pysik and G. Waidmann \\ Institut für Plasmaphysik, Forschungszentrum Jülich GmbH., Association EURATOM-KFA, \\ 52425 Jülich, Germany \\ G. P. Ermak \\ Institute of Radiophysics and Electronics of the Ukraine, Ak. Proskura st. 12, Kharkov 310085, Ukraine
}

(Presented on 10 June 1998)

\begin{abstract}
Progress in fusion research shows an increasing demand for diagnostics with high temporal and spatial resolution in order to study small and fast phenomena in fusion plasmas. Therefore, a new ten-channel pulsed radar reflectometer is under development for the TEXTOR Tokamak, to measure electron density profiles in the range of $0.4-4 \times 10^{19} \mathrm{~m}^{-3}$ at a high repetition rate of $2 \mathrm{MHz}$. An additional feature of this diagnostic is the possibility to perform correlation measurements at 10 $\mathrm{MHz}$ repetition rate to investigate density fluctuations. The reflectometer will scan the plasma with 1-ns-long radar pulses in the $18-57 \mathrm{GHz}$ range. In spite of the long flight time of the pulses of about $100 \mathrm{~ns}$, the above mentioned high repetition rates could be achieved by time multiplexing the generation and reception of the radar pulses. Temporal selection of the received pulses is performed by fast switching of the local oscillators inputs of the heterodyne receiver, to generate time windows in which the reception of the reflected pulses is expected. An embedded VME controller will manage the system and store the data with a speed of $20 \mathrm{Msamples} / \mathrm{s}$, up to a maximum of $64 \mathrm{Mbyte}$ data per plasma shot. In order to facilitate the handling of such a huge amount of data, an advanced data reduction scheme is being developed. Remote operation with a fast data link from FZ Jülich (Germany) to FOM Nieuwegein (The Netherlands) will be possible. (C) 1999 American Institute of Physics. [S0034-6748(99)68001-7]
\end{abstract}

\section{INTRODUCTION}

In pulsed radar reflectometry, short microwave pulses with a width in the order of $1 \mathrm{~ns}$ are launched into the plasma. ${ }^{1,2}$ Depending on the radar frequency and the plasma parameters, the pulse is reflected by a critical density layer and received again by the diagnostic equipment. The basic quantity that is measured by the pulsed radar diagnostic is the flight time of the microwave pulse between transmission and detection.

With the new setup, an upgrade of the four-channel pulsed radar developed by Heijnen, ${ }^{3-5}$ the number of channels is increased to ten. Moreover two additional variable frequency channels are added to the system. The latter two channels can be used in combination with two fixed frequency channels to perform correlation measurements and to study magnetohydrodynamic (MHD) modes. ${ }^{6}$ The pulse repetition frequency has been increased by a factor 10 in the upgraded system. The flight time is recorded with an accuracy of $70 \mathrm{ps}$, corresponding to a spatial resolution of $1 \mathrm{~cm}$ when reflected from a metal mirror in vacuum. The chosen pulse length of $1 \mathrm{~ns}$ is a compromise between the accuracy of the time of flight measurement and the pulse broadening, which would become unacceptably large for much smaller pulse widths. The flight time is measured between the 50\% levels of the leading edges of the transmitted and received pulse.

\section{MICROWAVE HARDWARE}

The outline of the microwave system of the TEXTOR pulsed radar reflectometer is given in Fig. 1. The system consists of three main parts:

(1) two homodyne channels at 18 and $24 \mathrm{GHz}$ in $K$ band waveguide,

(2) four heterodyne channels at $29,33,36$, and $39 \mathrm{GHz}$ in $K a$ band waveguide,

(3) four heterodyne channels at $47,51,54$, and $57 \mathrm{GHz}$ in $U$ band waveguide.

The $K a$ and $U$ band frequencies are alternately used as rf and local oscillator (LO) pulse. After mixing, all the heterodyne channels give an $18 \mathrm{GHz}$ intermediate frequency (IF) pulse. Two channels with variable frequencies around 36 and $51 \mathrm{GHz}$ are added to the system to perform correlation measurements with the corresponding fixed frequency channels.

The microwave sources are Impatt oscillators with an output power of approximately $200 \mathrm{~mW}$ in the $K$ and $K a$ band, and $100 \mathrm{~mW}$ in the $U$ band. All sources have an indi- 

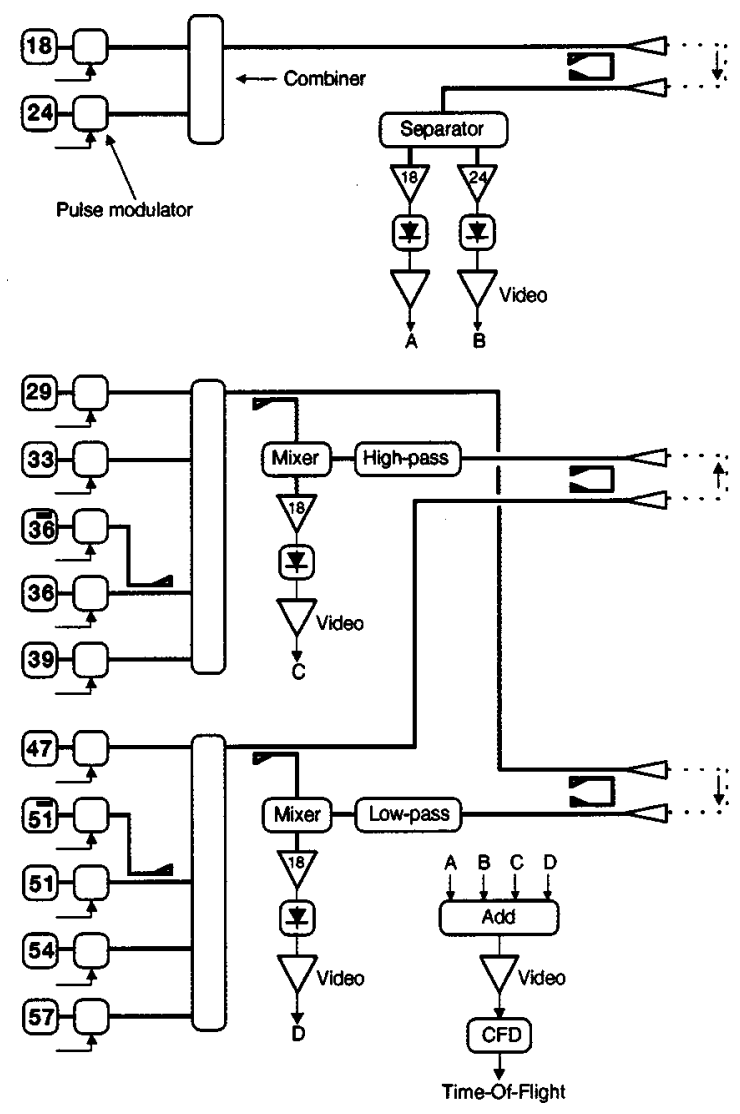

FIG. 1. Block diagram of the ten-channel pulsed-radar diagnostic, extended with two channels for correlation measurements. All frequencies are in $\mathrm{GHz}$. The sweepable sources are indicated with a minus sign.

vidual pulse forming modulator, which consists of a circulator and a varactor diode. The insertion loss of the modulators varies from 1.2 to $2 \mathrm{~dB}$. The isolation for the lower frequencies $(29-47 \mathrm{GHz})$ is approximately $30 \mathrm{~dB}$ and for higher frequencies $(51-57 \mathrm{GHz})$ it is $25 \mathrm{~dB}$. The modulators need a driver voltage of 5-10 V.

For both the 1-ns-wide rf pulse and the 20-ns-wide LO pulse, special drivers have been constructed that can produce pulses with a repetition frequency of $20 \mathrm{MHz}$. The 1-ns-wide pulse is Gaussian shaped. The pulsed LO selects a certain time window in which the heterodyne receiver is active. The output voltages of the LO driver pulses are individually adjustable, which gives the possibility to tune the amplitudes of the IF pulses to have approximately the same level at the video detectors. The drivers for the 1 and $20 \mathrm{~ns}$ pulses are combined in a single device with a $50 \mathrm{ohm}$ output impedance and a dc level control. With this level control $(0$ to $-1 \mathrm{~V})$ the various modulators can be tuned to the maximum isolation in the off state.

The channels in the three different sections are merged by means of combiners. The combiners consist of resonant cavities and circulators. As an example, the $K a$ band combiner is shown in Fig. 2. The main advantage over conventional directional couplers is the lower insertion loss of approximately $1 \mathrm{~dB}$ for the $K a$ band and $3 \mathrm{~dB}$ for the $U$ band. The frequency bandwidth for the different channels is approximately $2 \mathrm{GHz}$.

For the $K$ band system homodyne detection is chosen

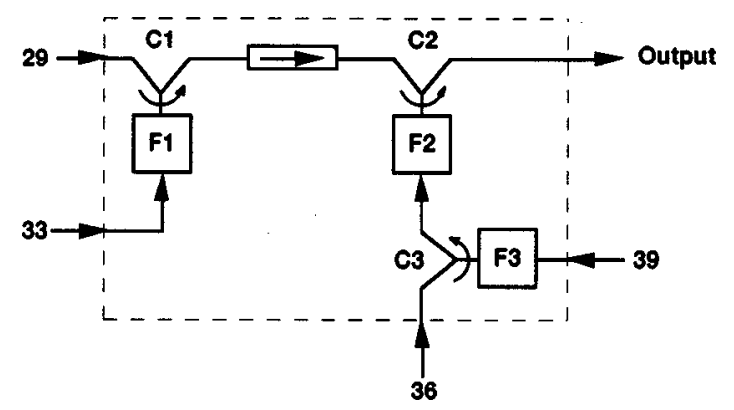

FIG. 2. Schematic of the $K a$ band combiner to merge the 29, 33, 36, and 39 $\mathrm{GHz}$ channels. $\mathrm{C} 1, \mathrm{C} 2$, and $\mathrm{C} 3$ are circulators. $\mathrm{F} 1, \mathrm{~F} 2$, and $\mathrm{F} 3$ are filters.

because of the relatively high pulse power of $250 \mathrm{~mW}$ and the lower waveguide losses as opposed to the $K a$ and $U$ band systems. Frequency selectivity is obtained with a frequency sensitive separator in combination with $1 \mathrm{GHz}$ wide 18 and $24 \mathrm{GHz}$ amplifiers. In the $K a$ and $U$ band systems, heterodyne detection takes care of frequency selective reception. The IF amplifiers in the two heterodyne systems have a gain of approximately $30 \mathrm{~dB}$ and a noise figure of $4 \mathrm{~dB}$. Detection is performed with Schottky barrier detectors. A simple antenna and waveguide system is chosen using separate transmitter and receiver antennas and oversized $X$-band waveguides. Oversized waveguides are necessary to keep the losses and the pulse broadening as low as possible.

The distance from the antennas to the radar setup is about $10 \mathrm{~m}$. Two echo pulses will be received from each transmitted radar pulse. The first one is a start pulse travelling via the bypass and the second one is the stop pulse reflected at the critical density layer. Jitter in the electronics is irrelevant in this setup. The minimum time between the two pulses is $4 \mathrm{~ns}$ which is determined by the constant fraction discriminator (CFD). The longest time delay is obtained from reflections at the far wall of the tokamak vacuum vessel when the density rises to near the critical density $\left(n_{c}\right)$. The longest time delay observed with plasma densities near $n_{c}$ is about 6 ns longer than the time delay without plasma. Calibration is performed using the time-of-flight (TOF) of the reflected pulse at the far wall in the nonplasma case. The bypass consists of two $10 \mathrm{~dB}$ directional couplers, an attenuator, and a short section of waveguide. The position of the bypass must be chosen in such a way that the reception of the start and stop pulse coincide with the 20 ns LO pulse. Tuning is possible by adjusting the length of the bypass waveguide, and by adjusting the timing of the LO driver. The attenuator must be set such that the start and stop pulse have approximately the same power level. After detection, the four video channels (A, B, C, and D) are preamplified with presettable amplifiers. The four signals are combined and then amplified to a level of about $5 \mathrm{~V}$ and fed to the CFD. The two output pulses will start and stop the time-offlight counter, developed at Rijnhuizen using eight parallel gated counters. ${ }^{3,7}$ The data produced by the TOF counter is fed to a data acquisition system built in VME. The data handling, storage, etc., will be described in Sec. IV. 


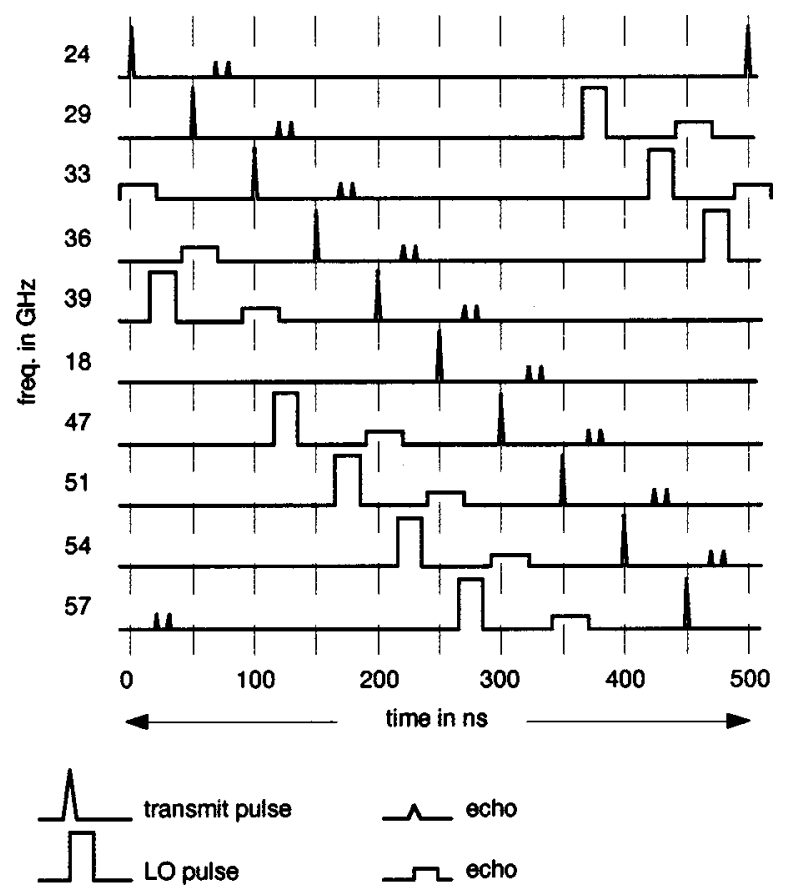

FIG. 3. Timing diagram for a ten-channel profile measurement.

\section{TIMING AND MEASUREMENT MODES}

A number of measuring modes are available. The first mode is a profile measurement with ten channels (see Fig. 3). The timing of each radar and LO pulse can be tuned individually by means of programmable time delay settings. During the existence of a LO pulse only one pair of matching start and stop pulses is present, which yields an $18 \mathrm{GHz} \mathrm{IF}$ signal (e.g., the $29 \mathrm{GHz} \mathrm{LO}$ pulse matches with the $47 \mathrm{GHz}$ radar pulses). False mixing may occur when a transmitted pulse acts as LO at a time an echo from the original $20 \mathrm{~ns} \mathrm{LO}$ pulse coincides in time. However, all these mixing products fall outside the bandwidth of the $18 \mathrm{GHz}$ IF amplifier. As an extra precaution a low-pass filter in the receiver of the $\mathrm{Ka}$ band, and a high-pass filter in the $U$ band receiver are added. The maximum measuring time, which is limited by the storage capacity of the $64 \mathrm{Mb}$ memory, is $3.2 \mathrm{~s}$ at a sampling frequency of $20 \mathrm{MHz}$. This $3.2 \mathrm{~s}$ time interval can be chosen freely within the TEXTOR discharge, which can last as long as $10 \mathrm{~s}$. It is also possible to run at a sampling rate of 10 or $5 \mathrm{MHz}$ and measure over 6.4 or $12.8 \mathrm{~s}$. Other measuring modes are possible when only one group of four channels is used. One measuring cycle will only take $200 \mathrm{~ns}$ in the four channel modes. Real quick firing of pulses occurs in the correlation mode, where only two channels are involved with a measuring cycle of $100 \mathrm{~ns}$.

For the correlation measurements two sweepable sources are available (36 and $51 \mathrm{GHz}$ ) with a sweep range of $1 \mathrm{GHz}$. The fixed and variable source alternately transmit a $1 \mathrm{~ns}$ radar pulse. These pulses are processed in the same way as in the profile measurements. During the discharge the variable source can be swept by tuning the driver voltage. This voltage is generated by means of an arbitrary wave generator (AWG). The number of frequency steps (max. 64), the num-

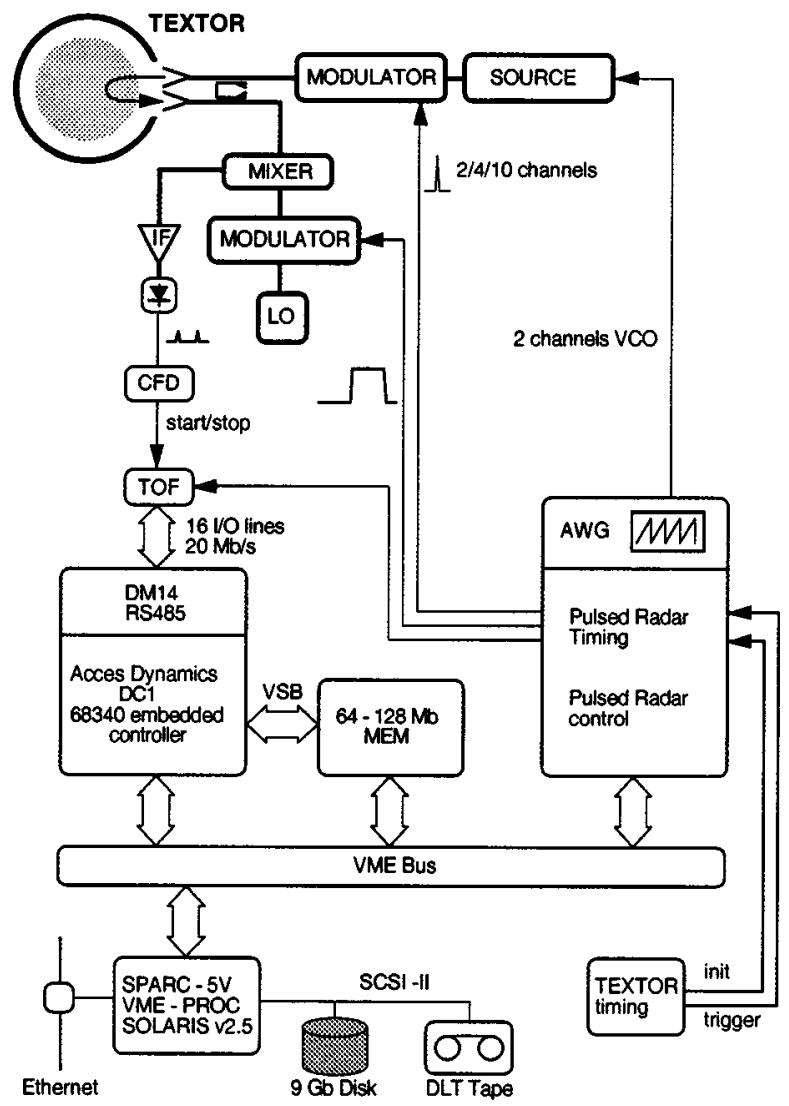

FIG. 4. Control, timing, data handling, and memory systems. Only one of the 12 microwave channels is given. For more details see Fig. 1.

ber of samples per frequency step, and the start and stop frequency can be set by computer.

\section{CONTROL, DATA HANDLING, AND STORAGE}

In order to facilitate fast control, data handling, and storage, the pulsed radar diagnostic is embedded into a VME system, operating under UNIX (Fig. 4). The output data of the TOF counter is stored by a DMA controller into a 64 Mbyte memory module. Data from this module can be addressed via VME by a SPARC CPU. With this computer architecture, the huge amount of data per TEXTOR discharge, can be processed locally. Local data reduction will become indispensable, in order to make the data transport to a distributed database and the interpretation of the measurements faster. The data acquisition software, which runs on this system, is based on commercially available software packages like Solaris v2.5.

The user of the pulsed radar diagnostic has access to the local VME system via Ethernet. The measurement mode, the status of diagnostic system, the settings of the AWG, the method of data processing, and data reduction, etc. can be controlled by the user by sending remote procedure calls to the SPARC CPU. Obviously, the system can be synchronized to TEXTOR. Besides, this ordinary measurement mode, the system can also run in a test mode, in which data is collected without TEXTOR synchronization and in which the hardware can be tested. 
Within the framework of the trilateral Euregio cluster (TEC) agreement between Germany, Belgium, and the Netherlands, it is envisaged that remote diagnostic operation with a fast data link from FZ Jülich to FOM Nieuwegein will become available in the near future. For this purpose a distributed database and communication software based on CORBA, for the communication between different software platforms like Windows NT and UNIX, is under development.

\section{CONCLUSION}

A ten-channel pulsed radar system with high repetition rates of $50 \mathrm{~ns}$ per channel has been constructed. The frequency range of the different channels is from 18 to $57 \mathrm{GHz}$. Two variable frequency channels at 36 and $51 \mathrm{GHz}$ are available for correlation measurements. The diagnostic has been installed at the TEXTOR Tokamak in Jülich, Germany. Remote control from FOM Nieuwegein will be possible. The first measurements can be expected in June 1998. Much work has still to be done in the development of intelligent data reduction schemes and data handling.

\section{ACKNOWLEDGMENTS}

The work described in this article was performed as part of a research program of the "Stichting voor Fundamenteel Onderzoek der Materie" (FOM) with financial support from the "Nederlandse Organisatie voor Wetenschappelijk Onderzoek" (NWO) and EURATOM. The Netherlands and Germany are partners in the Trilateral Euregio Cluster.

${ }^{1}$ C. A. J. Hugenholtz and S. H. Heijnen, Rev. Sci. Instrum. 62, 1100 (1991).

${ }^{2}$ V. F. Shevchenko, A. A. Petrov, V. G. Petrov, and U. A. Chaplygin, Plasma Study at T-11M Tokamak by Microwave Pulse Radar Reflectometer, Proc. 20th Conf. on Contr. Fusion and Plasma Phys., Lisbon, 1993, Part III, p. 1167.

${ }^{3}$ S. H. Heijnen, Thesis, University of Utrecht, Utrecht, 1995.

${ }^{4}$ A. J. H. Donné, S. H. Heijnen, and C. A. J. Hugenholtz, Fusion Eng. Des. 34/35, 73 (1997).

${ }^{5}$ S. H. Heijnen, M. R. de Baar, A. J. H. Donné, M. J. van de Pol, C. A. J. Hugenholtz, and the RTP team, Rev. Sci. Instrum. 66, 419 (1995).

${ }^{6}$ P. C. de Vries, A. J. H. Donné, S. H. Heijnen, C. A. J. Hugenholtz, A. Krämer-Flecken, F. C. Schüller, and G. Waidmann, Nucl. Fusion 37, 1641 (1997).

${ }^{7}$ M. J. van de Pol, S. H. Heijnen, and C. A. J. Hugenholtz, Proc. of the IAEA Technical Committee Meeting on Microwave Reflectometry for Fusion Plasma Diagnostics, JET, 1992 (unpublished), p. 228. 